\title{
New model for the hydroxyapatite-octocalcium phosphate interface
}

\author{
C. Zorrilla-Cangas ${ }^{2}$, M. E. Fernández ${ }^{1}$, R. García-García ${ }^{2}$, J. A. Ascencio ${ }^{3}$ and J. Reyes-Gasga ${ }^{2}$ \\ ${ }^{1}$ ININ, Km. 36.5 Carretera México-Toluca, 52045, Ocoyoacac Edo. de México, MEXICO \\ ${ }^{2}$ Instituto de Física, UNAM., A.P. 20-364, 01000, México, D.F., MEXICO \\ ${ }^{3}$ IMP, PIDD, Eje Central Lázaro Cárdenas No. 152, 07730, México D.F., MEXICO
}

Some experimental results have indicated that HA and OCP can form an epitaxial interface[1-3]. Hereafter the OCP-HA interface has become of great biological interest in the context of mineralized tissue formation. In order to reproduce the experimentally reported epitaxial growth between HA and OCP, the interface was formed joining half unit cell of HA and one cell of OCP, as it was indicated by Brown, but in such a way that $[000 \overline{1}]_{\mathrm{HA}}$ must be parallel to $[001]_{\mathrm{OCP}}$ and $[1 \overline{2} 10]_{\mathrm{HA}}$ is parallel to $[010]_{\text {OCP }}$ (Fig 1). From the free energy analysis in Brown's [4] model and in our model for the HAOCP interface it was found that in Brown's model, after relaxation, the atoms around the interface do not have the same environment as they have in the HA and OCP unit cells, whereas in our interface model they do. The P-O, Ca-O, and O-H distances in the zone around the interface were found in a reasonable range compare with those observed in the HA and OCP unit cells. All this indicates that our model is a better proposal for the study of the HA-OCP interface.

The HRTEM experimental image of the HA-OCP interface obtained by Iijima et al. [5](Fig.2) and the HREM simulated images obtained from our model show regions of good coherence in the interface separated by misfit-dislocation-like features. These structural defects are generated by the difference of $0.21 \AA$ between $\mathrm{a}_{\mathrm{HA}}$ and $\mathrm{b}_{\mathrm{OCP}}$ unit cell parameters. In the simulated image these defects are presented in a serie whose periodicity is of approximately 10nm. (Fig. 1 and 2)

The use of quantum mechanics calculations and the interpretation of electronic structure besides the crystals symmetry show a new way to determine the structure of interfaces, producing a whole supported model that must be considered in experimental studies. It is clear that the Brown's model was so good when the possibilities of understanding were limited to the geometry, however with the new developed tools, as the used in this work, it is possible to include electronic parameters in the solution of the structure, producing a well supported HA-OCP interface model.

\section{References}

[1] Iijima, M. \& Moriwaki, Y. (1998). J. Cryst. Growth 194, 125-132.

[2] Iijima, M. \& Moriwaki, Y. (1999). J. Cryst. Growth 198-199, 670-676.

[3] Iijima, M., Moriwaki, Y., Takagi, T. \& Moradian-Oldak, J. (2001). J. Cryst. Growth 222, 615-626.

[4] Brown, W.E. (1962). Nature 196, $1048-1050$.

[5] Ijima, M., Tohda, H. \& Moriwaki, Y. (1992)a. J. Crystal Growth 116, 319-326. 




Fig. 1. Serie of simulated HREM images for the interface model proposed in this work for different

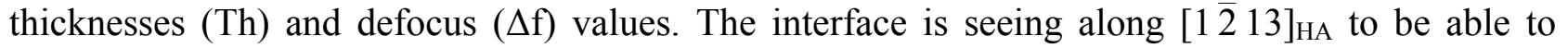
compare these images with the experimental image shown in figure 2 .

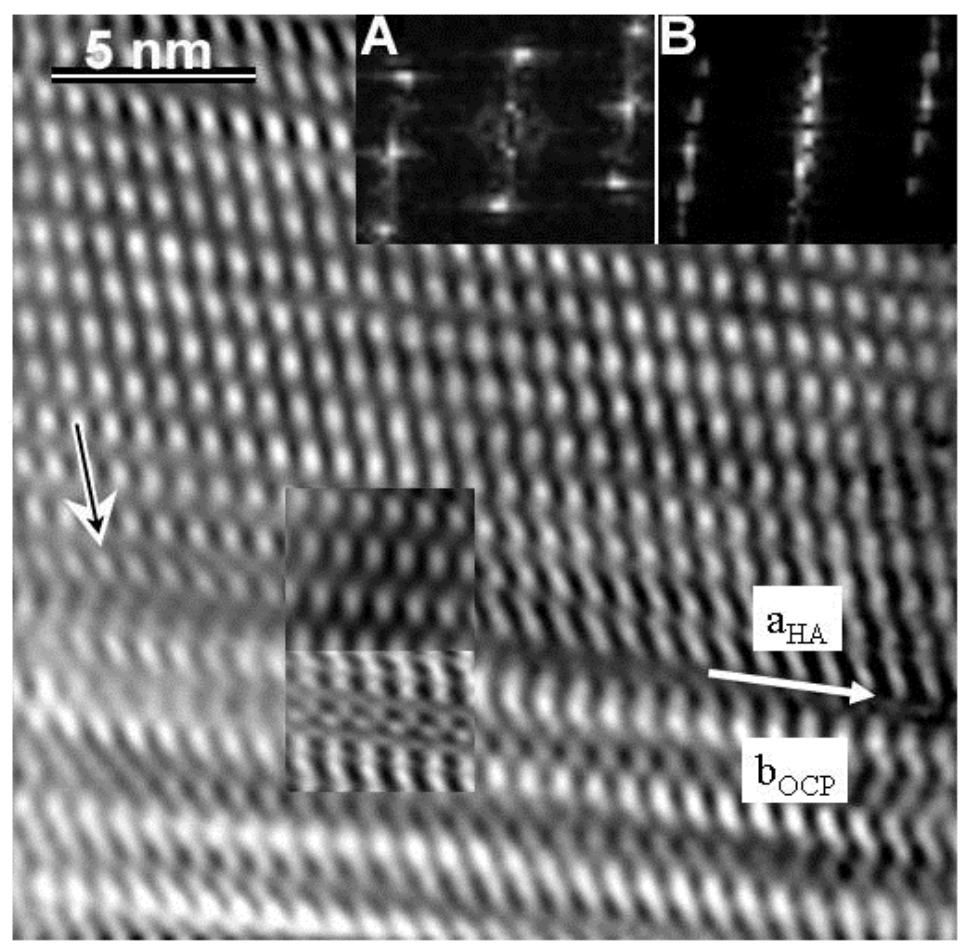

Fig. 2. Experimental HREM image along the $\left[\begin{array}{ll}1 & 13\end{array}\right]_{\mathrm{HA}}$ direction of the HA-OCP interface (Iijima et al. (1992a), used with authorization from the authors). a) Fourier transform from the HA zone. b) Fourier transform from the OCP zone. The simulated HREM image with defocus $\Delta \mathrm{f}=-550 \mathrm{~nm}$ and thickness $\mathrm{Th}=105 \mathrm{~nm}$ in the inset. Seeing this image at glanced incidence along the direction indicated by the arrow a misfitdislocation-like defect can be observed. 